\title{
Noncontact Extraction of Breathing Waveform
}

\author{
Yuzhe Zhang ${ }^{1,}$ a , Fei Shang ${ }^{1, b}$ \\ ${ }^{1}$ Department of Biomedical Engineering, Life Science School, Beijing Institute of Technology, \\ 100081, China \\ aemail: zyzbjlg@163.com, bemail:bit552sf@bit.edu.cn
}

Keywords: Breathing Waveform; Eulerian Motion Magnification; Principal Component Analysis; Video-based

\begin{abstract}
This paper presents a video-based method to extract breathing waveform from the upper body movement associated with breathing, which is based on Eulerian Motion Magnification and principal component analysis (PCA). Using our method, we are able to obtain the breathing waveform which contains different breathing pattern (normal breath, hold breath, quick breath, deep breath) exactly and rapidly from video without tracking the motion of body. Our method can also be used to extract the vibration pattern of other objects.
\end{abstract}

\section{Introduction}

Vital physiological signals such as heart rate, breathing waveform are closely related to our state of health and can be used to diagnose some common diseases. Traditionally, these signals are obtained only by some professional medical equipments. Recently, many new non-contact methods based on video or image sequence have been proposed to extract vital physiological signals. Such as the method used to extract heart rate based on ICA presented by Ming-Zher Poh et al. [1], the method used to extract pulse signal based on PCA presented by Guha Balakrishnan et al. [2] and the method used to extract breathing waveform based on a motion-tracking algorithm [3] presented by Dangdang Shao et al. [4].

We find that most of the video-based methods need to track the motion of the body, which is complex. In this paper we proposed a new simple video-based method to extract the breathing waveform rapidly and accurately by the camera of mobile phone or personal computers and it is very convenient for us to monitor our state of health.

Our method is based on Eulerian Motion Magnification [5] and principal component analysis (PCA). Eulerian Motion Magnification can be used to amplify and visualize small motions or subtle change of color in a certain frequency band from the standard video sequence, so it can be used to amplify the movement associated with breathing in the video. Then, principal component analysis (PCA) is used to denoise and extract the waveform exactly and robustly from the image sequence. The details about our method will be described in the next part. And the simulation results show the method is effective and can be realized in PC or mobile devices conveniently.

\section{Method}

Eulerian Motion Magnification algorithm is based on an Eulerian framework for fluid flow analysis, observing and manipulating temporal variations at fixed locations and it is very different from the traditional Lagrangian method which needs to trace the motion of objects [5]. The process of Eulerian Motion Magnification is as follows. Firstly, the video sequence is decomposed into different image sequences (Laplacian pyramid and Gaussian pyramid) at different scales [6]. And each image sequence at different scale contains different information. The value changing over time of each pixel at every image sequence is considered as a time-domain signal, then a bandpass filter is used to extract the interested information in particular frequency band from these signals, thus we can get some new image sequences only containing the information in particular frequency band. A new video can be reconstructed with these processed image sequences, the motion or color change 
which is difficult or impossible to see by the naked eye will be enhanced in the reconstructed new video.

When we breathe our upper body move up and down slightly, sometimes the movements are not noticed. In order to extract the breathing waveform, the Eulerian Motion Magnification algorithm is used to amplify the movement associated with breathing in source video which contains the upper part of body. We can get the Laplacian pyramid sequences and Gaussian pyramid sequences at different scales. The Laplacian pyramid sequences contain and highlight the edge of objects. The movements such as that associated with breathing can make the pixel values on the edge of the object change severely, which will show obviously in the Laplacian pyramid sequence. So the Laplacian pyramid sequences should be used to extract the breathing waveform. Laplacian pyramid sequence at different scales contains different movement so we must use the image sequences at appropriate scales (containing the movement associated with breathing) to extract breathing waveform. The movement associated with breathing is a kind of vertical movement. To extract the waveform of this vertical movement, we compute the average of all the pixels values in an each row in each image of the Laplacian pyramid sequence. The average changing over time of each row is considered as a time series. Then we process these $\mathrm{N}$ (the number of rows of the image sequence) sets of time series using PCA algorithm (We assume that the noise and the breathing waveform satisfy Gaussian distribution) and the first principal component is the final output, the breathing waveform. Fig. 1 shows the whole process of our method.

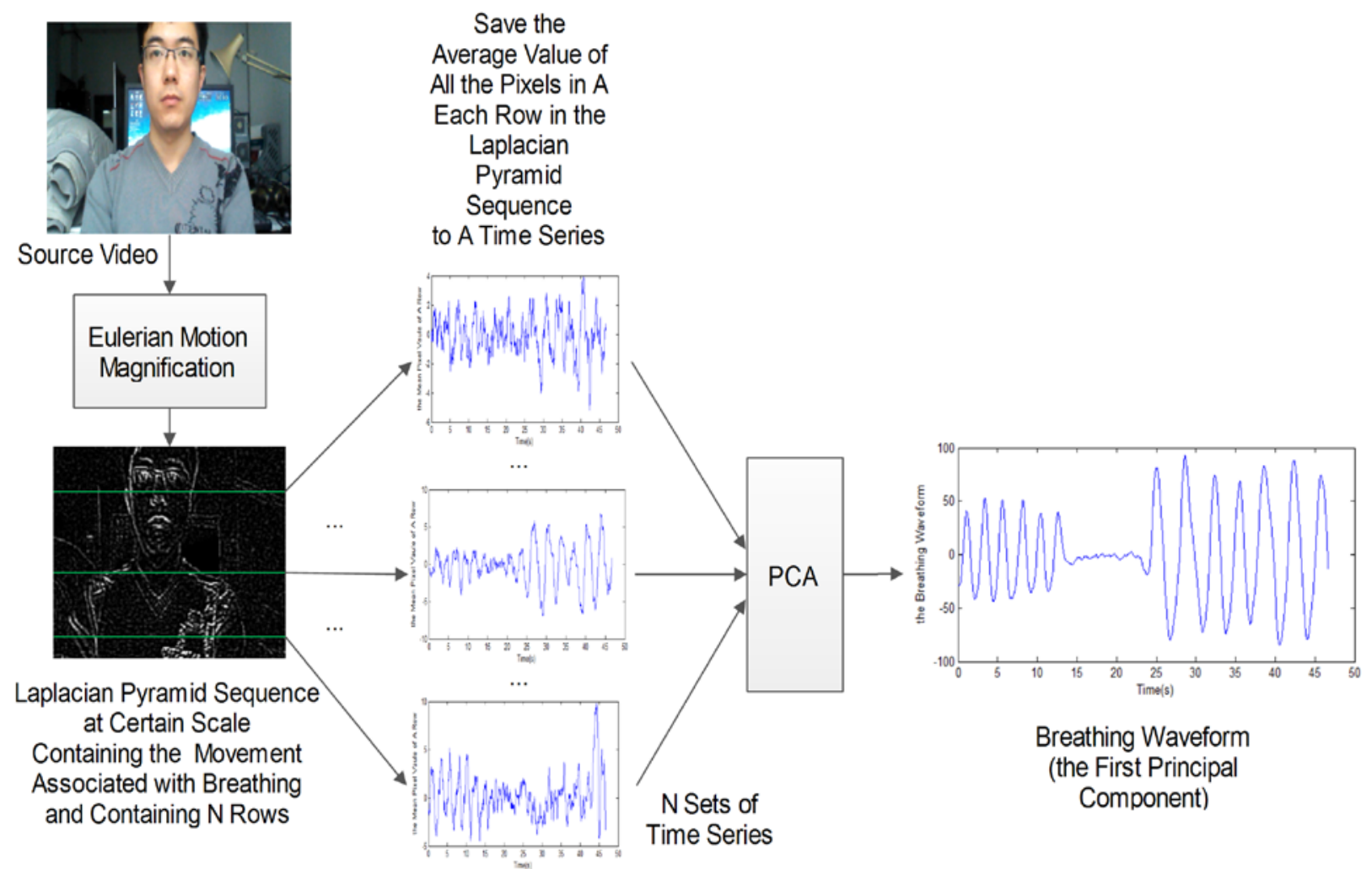

Fig.1. The process of our method to extract breathing waveform.

\section{Experiments and Discussion}

We record a video containing the upper part of body with the camera of mobile phone or personal computers (in this paper we used the front-facing camera of Redmi). During the recording process, the person sit still and the distance between camera and face should be about $50 \mathrm{~cm}$ to $70 \mathrm{~cm}$, it is a reasonable assumption for people using computer. To reduce computational complexity we resize the source video to $512 \times 384$ then convert the video which contains three channels $(\mathrm{R}, \mathrm{G}, \mathrm{B})$ to gray image sequence. Then we should extract breathing waveform from the gray image sequence. 
Moreover, it is important that the video must contain the chest and the region of chest in the video with 384 rows should be more than 128 rows (about 1/3 of the rows of the image) (see Fig. 2).

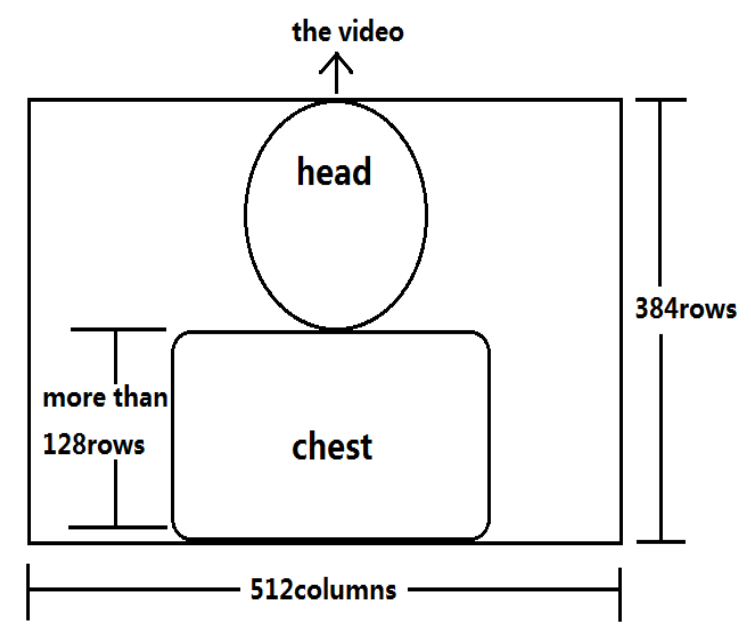

(a)

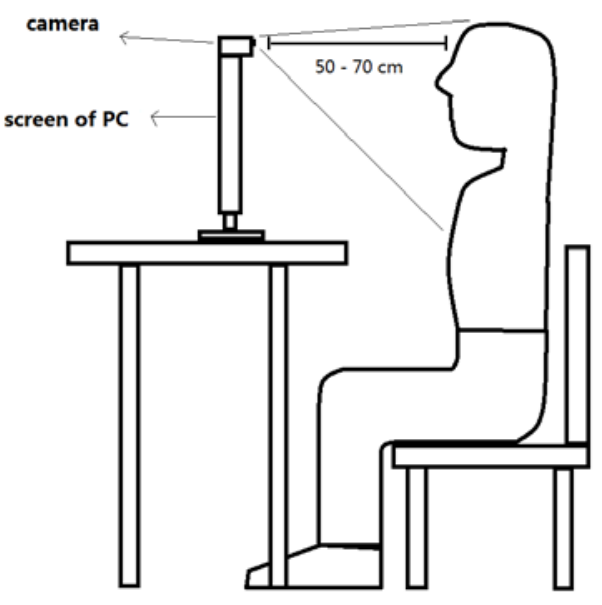

(b)

Fig.2. (a) Schematic illustration of source video containing the upper part of body used to extract the breathing waveform. (b) Schematic illustration of experimental setup.

Our method has two important parameters in the process of Eulerian Motion Magnification, the band-pass frequency of the filter to amplify the movement associated with breathing, and the scale of the Laplacian pyramid.

The breathing rate of a healthy person is about 14 to 20 times per minute and the corresponding frequency band is 0.2 to $0.33 \mathrm{~Hz}$. So a Butterworth filter (By using the Butterworth filter the result of the Magnification of the breathing movement can be shown in real time [5].) with the band-pass frequency $0.2 \sim 0.6 \mathrm{~Hz}$ will be used to filter the Laplacian image sequence and amplify the movement associated with breathing.

In order to find the optimal scale of the Laplacian image sequences used to extract breathing waveform, we extract the waveform (the first principal component obtained from the process of PCA) at six different scales (sigma $=1,2,3,4,5,6)$ with our method. The result is showed in Fig. 3. The breathing waveform extracted from this video should contain three breathing patterns, normal breath, hold breath, and deep breath. We find that the waveform extracted from second-scale Laplacian image sequence is better than others. In these image sequence the higher the scale is, the more information about breathing is lost, and the lower scales contain more noise and the size of image is larger, it will cost more time to process the image sequences at lower scales. We select the second scale as the optimal scale to extract breathing waveform. But if the distance between camera and face is different or the quality of the video is different, the optimal scale will be different. So the appropriate scale must be selected first by experiments, when our method is used

When the source video were recorded, the participants were asked to maintain four different breathing patterns, normal breath for 15 seconds, hold breath for 5 seconds, quick breath for 15 seconds and deep breath for lasting 15 seconds. Fig. 4 shows an example of the extraction of breathing waveform with the optimal parameters. Fig. 4 (a) is one frame of the source video. Fig. 4 (b) is one frame of the Laplacian pyramid sequence at second-scale. The breathing waveform extracted from the source video is shown in Fig. 4 (c). We can see that there are 4 different breathing patterns, normal breath, hold breath, quick breath and deep breath in the waveform. The results show the breathing waveform is extracted accurately. 


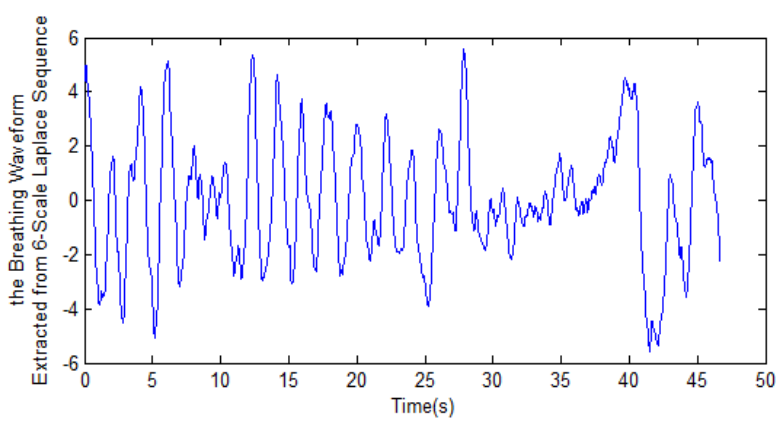

(a)Sixth-scale (sigma=6)

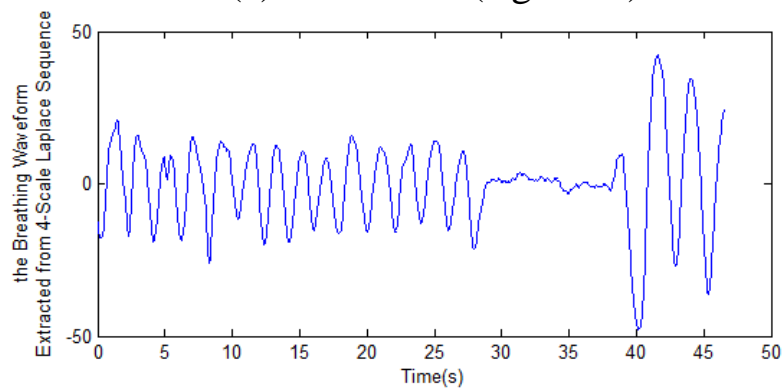

(c)Fourth-scale (sigma=4)

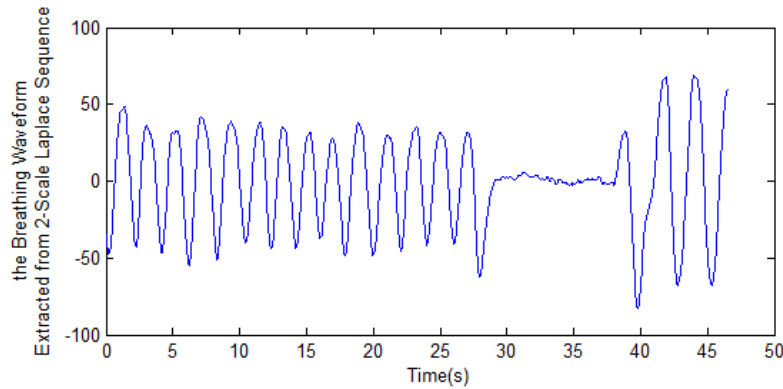

(e)Second-scale (sigma=2)

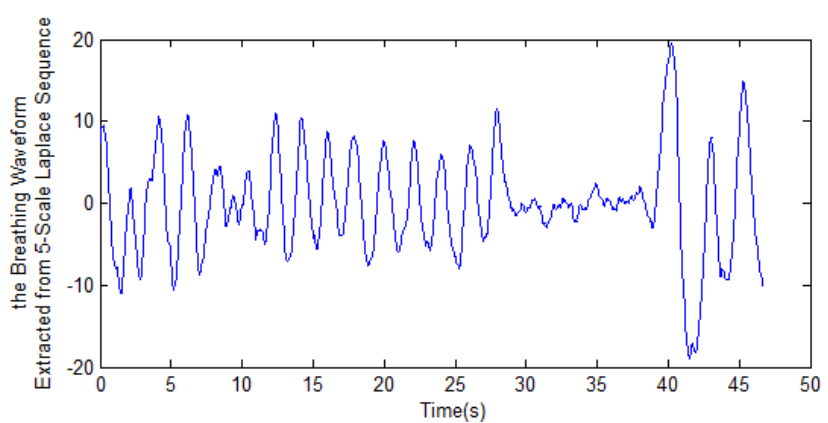

(b)Fifth-scale (sigma=5)

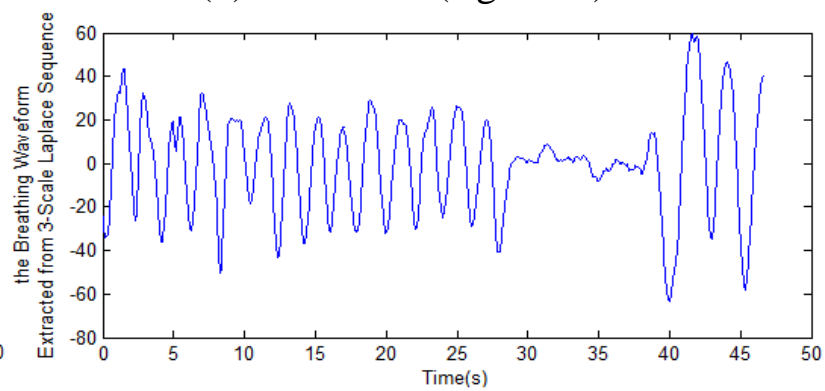

(d)Third-scale (sigma=3)

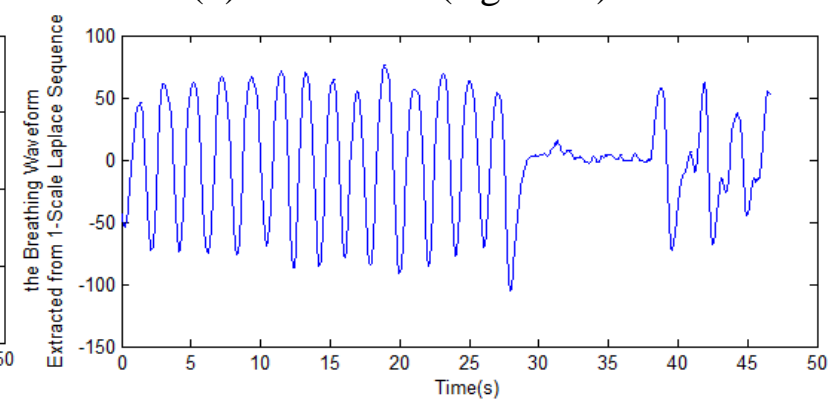

(f)First-scale (sigma=1)

Fig.3. The breathing waveforms extracted from different Laplacian image sequences at different scales. (a) to (f) correspond to the waveforms extracted from 1 to 6 scale Laplacian image sequences.

The method occupies less than 4M RAM, so it can run on almost every mobile phone and computer. And it will cost about $15 \mathrm{~ms}$ to process one frame of the second-scale Laplacian image sequences on our PC with a four-core processor and 8 GB RAM. The frame rate of the video we used to extract breathing waveform is $30 \mathrm{fps}$ (The corresponding sampling interval of the camera is about 33ms). So our method runs in real time on our PC. Because the frequency of our breath is less than $1 \mathrm{~Hz}$, we can reduce the sampling frequency to $10 \mathrm{~Hz}$ (The corresponding sampling interval of the camera is about $100 \mathrm{~ms}$ ). Thus our method can be transplanted into most of the mobile phones and computers to extract the breathing waveform in real time.

Besides the breathing waveform, the vibration patterns of other objects can also be extracted with our method. The appropriate band-pass frequency and scale should be determined firstly according to the movement characteristics of the object. Fig. 5 shows an example of the extraction of the hand horizontal vibration.

In this example, the hand vibrates horizontally and slightly (The amplitude is less than $1 \mathrm{~cm}$ ) and the vibratory rate is 60 to120 per minutes. To extract the waveform, we use a Butterworth filter with the band-pass frequency $1 \sim 2 \mathrm{~Hz}$ to filter the Laplacian image sequence and we choose the fourth-scale Laplacian image sequence to extract the waveform. The vibration of the hand is a kind of horizontal movement, so we consider the average changing over time of each column in the image sequence as a time series. Then we process those time series with PCA algorithm and we get the waveform shown in Fig. 5 (c) from the first principal component. 


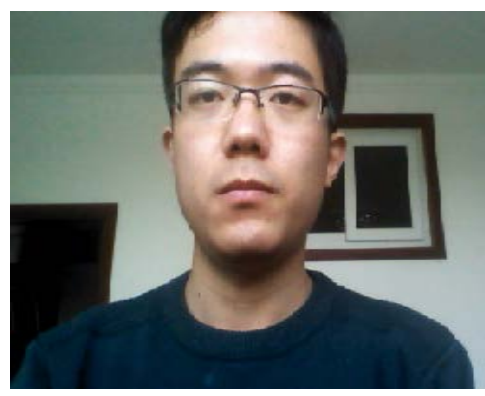

(a)

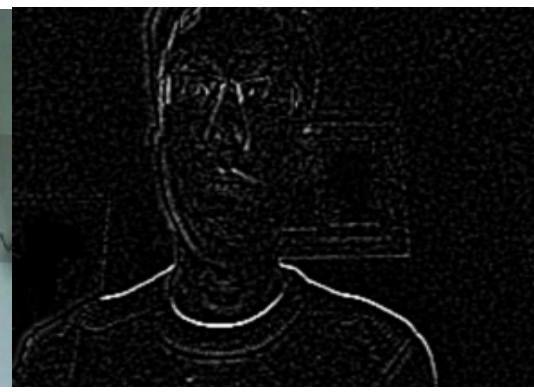

(b)

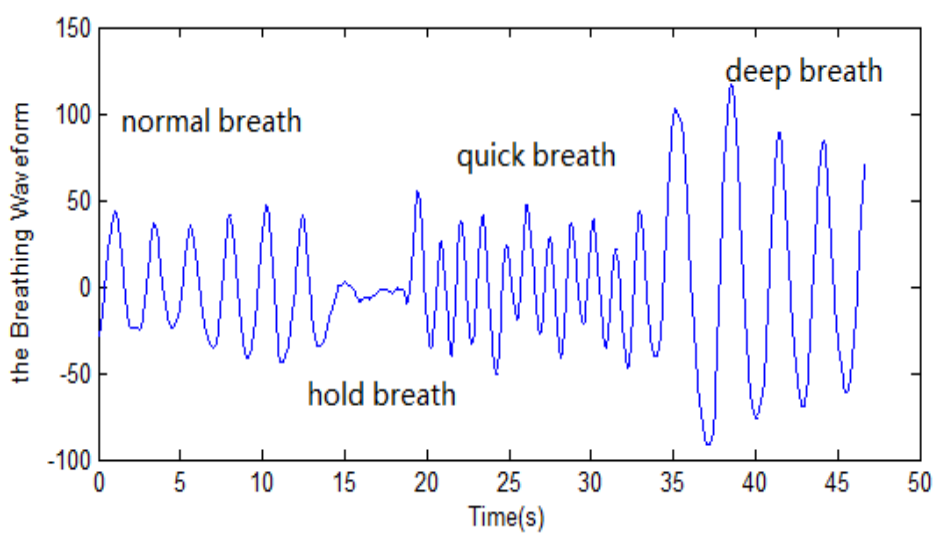

(c)

Fig.4. The results of the extraction of breathing waveform. (a) One frame of source video containing the upper body. (b) One frame of the second-scale Laplacian pyramid sequence. (c) The breathing waveform extract from the video which shows different breathing pattern (normal breath, hold breath, quick breath, deep breath).

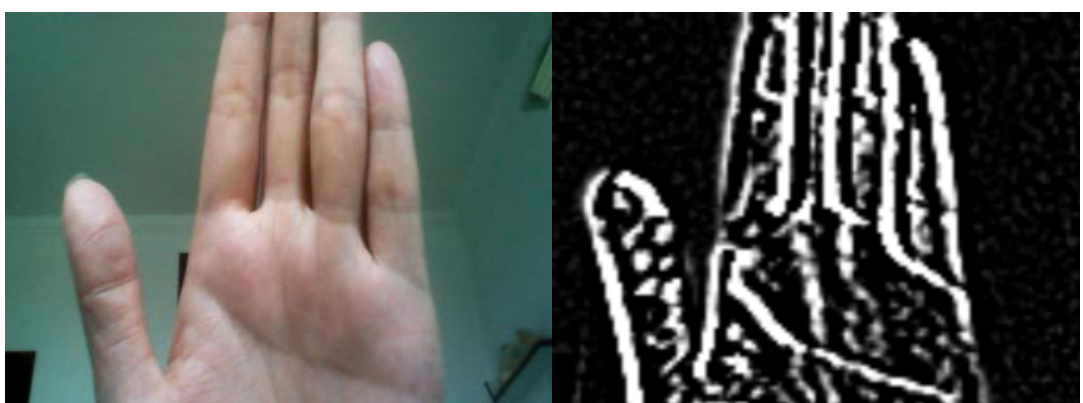

(a)

(b)

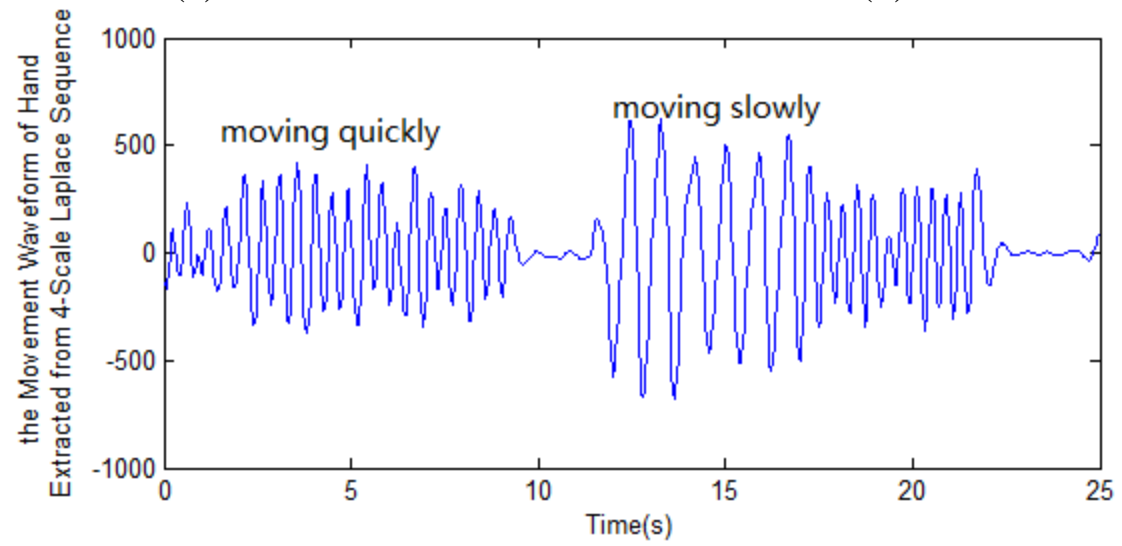

(c)

Fig.5. The results of the extraction of the hand horizontal vibration. (a) One frame of the source video containing the vibrating hand. (b) One frame of the forth-scale Laplacian pyramid sequence. (c) The waveform extract from the source video. 


\section{Conclusion}

Using our method, we can extract the breathing waveform from the video containing the upper part of body accurately. Our method is robust and it can satisfy the request of real time. The method is simple it can be transplanted into the different platforms, such as mobile phones or PC easily. It is a convenient way to monitor our breath state. But our method has an assumption that people have no obvious movement, and it limits the application of our method.

\section{References}

[1] Poh M Z, McDuff D J, Picard R W. Non-contact, automated cardiac pulse measurements using video imaging and blind source separation [J]. Optics express, 2010, 18(10): 10762-10774.

[2] Balakrishnan G, Durand F, Guttag J. Detecting pulse from head motions in video [C]//Computer Vision and Pattern Recognition (CVPR), 2013 IEEE Conference on. IEEE, 2013: 3430-3437.

[3] Jin S, Koh G. A robust image tracker based on phase correlation and Fourier-Mallin transform [C]//Control, Automation and Systems, 2008. ICCAS 2008. International Conference on. IEEE, 2008: 1028-1031.

[4] Shao D, Yang Y, Liu C, et al. Non-contact monitoring breathing pattern, exhalation flow rate and pulse transit time [J]. IEEE Transactions on Biomedical Engineering, 2014, 61(11): 2760-2767.

[5] Rubinstein M. Analysis and visualization of temporal variations in video [D]. Massachusetts Institute of Technology, 2014.

[6] Burt P J, Adelson E H. The Laplacian pyramid as a compact image code [J]. Communications, IEEE Transactions on, 1983, 31(4): 532-540. 